that these pains, which tend to arise in a less acute manner, are also generally of the focal type.

5. The final suggestion is therefore that the myalgic spots which it is now widely agreed form the basis of many of the rheumatic syndromes of later life are sometimes a legacy from acute infections in earlier life. It is important to remember that the victims are often unaware of their presence until they become activated by factors such as chill, trauma, or focal sepsis. These factors should therefore be regarded as secondary and no longer causative in such cases.

My thanks are due to the D.M.S. PAIFORCE and to Maj.-General R. Barnsley, M.C., D.D.M.S. Southern Command, for permission to publish this paper, and to Dr. H. Jamieson, C.M.O. of the A.I.O.C., for help.

\title{
NEW PATHS OF INVESTIGATION FOR DIAGNOSIS AND THERAPY
}

\section{BY DR. ERNST FREUND}

Strck 1828, when Woehler discovered a method of producing urea in the test tube, thereby proving that some of the substances of organic life could be produced in vitro, pathological investigation has progressed towards a more precise understanding of the activity of the human organism in the sphere of biochemistry.

The study of chemical phenomena, aiming at qualitative and quantitative determinations of the waste products of metabolism, was thus encouraged with a view to the possibility of distinguishing between pathological and normal metabolism, and of this becoming the basis of diagnosis and therapy. There can be no doubt that such a development offers considerably better prospects of effective therapy than the empirical employment of substances of the mineral, plant and animal worlds, dating from the earliest age of medicine (admittedly with very beneficial results in some aspects of the art of healing).

Progress in development must necessarily be sectional. A complete survey of the vast range of biochemical problems involved in the utilisation of varied substances by the human organism, and the waste products of the process of utilisation, is a task of such magnitude that it can be only usefully approached little by little. This paper will deal with certain aspects of diagnosis and therapy arising from a comparison of the effects on the cells of material in the urine coming respectively from $(a)$ healthy and $(b)$ morbid subjects.

We can easily ascertain how much of the ingested food is employed 
in the body; how much is used up in producing heat and activity. We can note whether this is occurring in a normal manner, or whether anomalies attributable to deficiency in the organs are occurring. Indeed, in a number of cases we are able to determine, by means of "load tests," the capacity of some organs. But the question, "Is a serious illness present ?" which is so important both to the doctor and the patient, is only rarely answered by evident deficiency in an organ. But the promising field is being opened up of biochemical investigation of the "waste products" eliminated by the various organs.

Let us picture the conditions prevailing in an organism or in the cell of an organism. Its chemical constituents, its store of substances, are subject to continuous change; and change can be intensified or reduced by the action of heat and cold, or by the ingested food, or even by the breathing - that is to say, by external reagents. Also, apart from these reactions, the cell of the organism is subject to influences which from youth to a certain age may be constructive, and at a later age may be destructive. There can be no doubt that the constructive and destructive processes may proceed side by side in the cell. But so far research has concentrated chiefly on katabolism. Under the impression that katabolism is incomparably greater than the anabolism that must be ascribed to the tissues, investigators have studied chiefly the former, regarding the latter as secondary, because it could only be governed from the cell, which takes from the sea of nutrient juices flowing to it the substance which the miracle of selectivity enables it to take.

Now what happens when the organism is ill? In some cases the organs lose weight, become emaciated, so that parts of the cells must have been destroyed. In other cases the cell is diverted from its right functions of maintenance and of normal growth and there follows the creation of superfluous or malignant tissue. What effects this morbid process and in what circumstances? The answer is-apart from the natural degenerative conditions of "wearing out" by age - external enemies, either of a physical character, as heat and cold, or microorganisms.

Consider the physical conditions first. The initial effect of heat or cold on a particular group of cells coming into contact with them does not finish the matter. That effect may spread to other cells not directly involved. This compels the assumption that destructive ferments have been set in motion (and this can be demonstrated on living tissue by means of autolytic experiments). Where do these ferments come from?

We know that the most important digestive ferment of the intestinal canal, trypsin, becomes inactivated on entering the serum, and that it may be partially reactivated through slight acidification. On the 
basis of this example it is possible to postulate that each cell contains constructive and destructive ferments, which can be inactivated when linked with each other or with other substances, and can be activated by certain stimuli. Thus the effect of an external attack on a cell will depend on which of the ferments is liberated, and which, when already liberated, can be inactivated again, or eliminated from the cell and the organs. To throw light upon these questions it is of particular value to examine the urine for constructive or destructive ferments, and to see whether there is any difference in the action of those ferments on added tissue extracts in a state of health and illness respectively. The investigation was to be not of digestive ferments, but ferments for cell components. It was therefore of importance to discover, in the first place, whether ferments destructive of the nucleus of the cell were present (nucleases). For this purpose in a series of experiments I prepared alkaline extracts of an organ, precipitated out the nucleoproteids with acetic acid, and used the nucleoproteid solution which remained in the form of a cloudy solution after the acetic acid had been washed out as a reagent. I used $0.05 \mathrm{~g}$. per cent. $\mathrm{N}$ of this nucleoproteid, adding 1 to 10 c.c. urine, then alkalising to approximately pH 8.5, so that phenolphthalein did not turn pink, adding one drop of chloroform, and leaving the mixture to stand at a temperature of $37^{\circ}$.

After ten hours it could be noted that healthy urine destroyed this nucleoproteid to such an extent that no turbidity, or only traces, could be obtained with acetic acid. But the urine of a person with a sick organ had not only lost its power of solvency for the nucleoproteid of that organ, but had often acquired an anabolic power for it.

As a complement to this finding, investigation of extracts from healthy and morbid organs showed that in the case of healthy organs added peptone exerted a constructive action, while in the case of morbid organs it exerted a destructive action. Thus we have the following conclusion: in the healthy organs constructive ferments for the cell-extract substance are at work; in the morbid organ destructive ferments; and as the examination of the urine shows, the healthy organ sends destructive ferments into the urine, while the morbid organ is incapable of this and, as far as can be ascertained, sends constructive ferments into the urine.

These facts have been completely confirmed in urine examinations repeated a thousand times (see also confirmations by Bume, Wr. klin. Woch., 1936, 46, and Lustig, Klin. Woch., 1937, 2). The differentiation goes so far that it is possible to distinguish between a disease of the small and large intestine. The corresponding ferment actions of the serum are accordingly of a contrary kind to those of the urine. This opens up the possibility of supplementing the knowledge we are able to acquire through auscultation and percussion with the study of the chemical functions of the individual organs regarding their cell material. The 
reaction on the nucleoproteids naturally represents only the beginning of this study, which must be extended to all the other great groups of cell substances as fats, carbohydrates, lipoids, etc., and to the extent of this constructive or destructive process, so that the kind of organ destruction may be determined and also whether the products involved are, functionally, cardinal or secondary products.

Thus, to complement the diagnosis (e.g., in the case of hypertrophies), the urine result may be compared with the construction or destruction of the serum, and the quantitativity of the reaction may be investigated.

However, from the point of view of diagnosis it will not be sufficient to know that an organ is diseased; it will also be necessary to determine the kind of disease. We must know whether it is tuberculosis, or a purulent disease, or degeneration, etc.

There is a possibility that the sick organ will show a stronger inhibition to katabolism than the normal organ. To investigate this it would be necessary to proceed in the following manner:

Take the case of a rheumatic joint. We can prove that the joint is morbid, but we do not know whether the morbidity is rheumatic in origin or otherwise. It will be necessary to submit to digestion another organ, say muscle, which in the healthy state is normal for the urine concerned, in order to discover whether constructive or destructive action takes place. In some cases it will perhaps be necessary to mix a healthy organ with the toxin concerned and thereby poison it. In this way it would be possible - and past experiments have shown this possibility - to diagnose the kind of illness as well.

However, as to the question whether a grave illness is present, it is not necessary to make tests for all organic illnesses; it is sufficient, as investigations have shown, to test the nucleoproteid of the serum as a sort of representative of the entire organism - with the urine.

However, diagnosis is not the ultimate aim, only an aid to the ultimate aim of therapy. But this study also offers a new possibility for therapy.

It is a medical view dating back to antiquity that the organ that is sick should be fed with the same organ substance. The modern method of injections of extracts aims at this therapeutic action. But the urine reaction in question-that is, the testing of the functions of the organs as regards construction and destruction - affords a further possibility of extract of precisely the right substance. If the healthy organism sends the destructive ferments into the urine and retains the constructive ferments, then, by preparing extracts from the organ, it must be possible to determine the substance which acts particularly constructively - that is, the one which, when added to the urine, impedes the destructive process.

This determination can be carried out by adding to the normal 
urine, which acts destructively, the organ extracts, and determining the one that impedes the destructive action. This organ extract, after being isolated and concentrated, would have to be tested for its therapeutic action. Possible therapeutic action in this respect would possess the advantage over other therapeutic measures with injections of organ extracts. Up till now the functional products, so to speak, have been injected, so that only a replacement of the original product is effected, whereas the injection of constructive substances would not only normalise the organ, but also increase its normal productivity. This effect could be proved by means of perfusion experiments.

Steyskal in Vienna has already reported on a number of therapeutic effects in kidney cases which he obtained with organ ether extracts which I have supplied.

All these suggestions are merely an indication of a path that has yet to be explored. But when it is explored, the physician may be able to do for the sick organism what the healthy living cell does for itself through its most essential property - recruitment of its substance.

In the investigation of rheumatic diseases we have carried on at the Pearson Foundation some experimental work on lines indicated in this paper. This work was much hampered by lack of sufficient clinical material which would enable tests to be carried out under control conditions.

The fact that if people are exposed to what are accepted to be factors promotive of rheumatic disease (such as damp and cold) all do not develop rheumatic symptoms points to the assumption that these are due not only to such external stimuli, but also to predisposition or metabolic anomalies.

In search of such anomalies, we investigated whether rheumatic urine behaved differently from non-rheumatic urine in the presence of joint extracts. We found that, whereas normal urine digests added nucleoproteid of normal joints at $37^{\circ} \mathrm{C}$. to such a degree that no clouding can be obtained with acetic acid, rheumatic urine left added nucleoproteid completely unchanged.

The diagnostic application of this reaction proved itself to the extent that an improvement of the rheumatic condition was indicated by the fact that the patients' urine had recovered its power to digest nucleoproteid from joints. True, this only proved a morbid state in the joint, not necessarily a rheumatic state.

War conditions prevented us from obtaining rheumatic joints for such diagnostic investigation. Therefore we tried to produce "rheumatic substances," so to speak, by poisoning normal joint extracts with extract substances from rheumatic fæces. It had been ascertained from earlier investigations that if, at the onset of the disease, rheumatic fæces were inoculated into superfatted milk, a bacterium-free extract could be obtained from the milk, which, if 
injected into mice, caused swellings of the joints of the hind legs. This reaction was evidently due to special substances in the food, for the same process with peptone solution produced no such effect. Extracts of this "rheumatised" milk were applied to joints or joint extracts, and thus there was obtained from the joints nucleoproteids which, while digested by normal urines, remained undigested by rheumatic urines. This indicated a possibility of the diagnosis of the rheumatic state.

Further investigations showed that the "toxic" substance is a fatty acid which can be isolated as a calcium salt and which, if injected into the neck of mice, causes difficulty of movement in the joints of the hind legs.

As regards therapy, these results suggest the conclusion that abnormal acids exerting a harmful action on the membranes of the joints are produced in the intestinal canal through abnormal fermentative processes and point to a therapeutic method of treating rheumatism by means of intestinal disinfection, with simultaneous alkalisation.

\title{
EMPIRE RHEUMATISM COUNCIL
}

\author{
SEVENTH ANNUAL REPORT \\ NOVEMBER, 1942, To NovEMBER, 1943
}

My Lords, Ladies and Gentlemen,

THE hope that this, the seventh completed year of our work, would allow members to assemble in Annual Meeting has been again deferred by war conditions, but we may have reasonable confidence of meeting in 1944. Meantime this report records the progress of the past year.

With deep regret I have to note the death of two of our VicePresidents, Lord Hirst of Witton and Sir Benjamin Cohen, both generous supporters of the Council; of Sir Francis Fremantle, M.P., and Dr. Geoffrey Holmes and Dr. F. J. Poynton, two of our keen scientific workers.

The Right Hon. Tom Williams, M.P., Parliamentary Sceretary to the Minister of Agriculture, has accepted office as a Vice-President of the Council, a welcome addition to our ranks. $\mathrm{He}$ is strongly interested in the War on Rheumatism, and this link with Agriculture will be valuable since wholesome diet is an important factor in dealing with rheumatic disease. 